Check for updates

Cite this: Mater. Chem. Front. 2019, 3, 151

Received 24th August 2018, Accepted 16th November 2018

DOI: $10.1039 / \mathrm{c} 8 \mathrm{qm} 00424 \mathrm{~b}$

rsc.li/frontiers-materials

\section{Rational design of aggregation-induced emission sensor based on Rhodamine B for turn-on sensing of trivalent metal cations, reversible data protection, and bioimaging $\dagger$}

\author{
Hai-Xia Yu, Junge Zhi, (D) * Zheng-Feng Chang, Tianjiao Shen, Wei-Lu Ding, \\ Xiaoling Zhang (D) and Jin-Liang Wang (D) *
}

\begin{abstract}
A novel AIE-active molecule (TPEThRB) composed of a tetraphenylethene unit, Rhodamine spirolactam, as a receptor, and thiophene ring as a conjugated electron-rich linker was obtained. It can be utilized to effectively detect trivalent metal cations and $\mathrm{H}^{+}$ions, accompanied with visible color change and turn-on fluorescence enhancement. The four species can be further recognized individually through UV-Vis analysis. Moreover, compared to the conventional manner of metal ion chelation-induced ringopening form of Rhodamine B derivative, the interaction of TPEThRB with trivalent cations is ascribed to the synergy of proton-induced open-cycle and the subsequent coordination interaction due to the steric effect and acidic hydrolysis of trivalent metal cations. In addition, in view of its acidochromism property with reversible color and fluorescence changes, AlE-active TPEThRB may be a prominent smart material for data protection by employing trifluoroacetic acid vapor as the chromogenic reagent and diethylamine vapor as the eraser. Owing to the AIE characteristics and the remarkable photophysical response characteristics towards trivalent metal ions in vitro, TPEThRB is suitable for fluorescence ratio imaging of trivalent metal cations in living cells.
\end{abstract}

\section{Introduction}

Due to the involvement of organic fluorescent materials in widespread applications, such as organic light-emitting diodes (OLEDs), fluorescent sensors, mechano-sensors and bioimaging, they have received significant attention. ${ }^{1}$ Considering the inherent sensing merits of visualization, sensitivity and selectivity, fluorescent molecules have played a great role in detecting various analytes in living organisms. ${ }^{2}$ Trivalent metal cations, $\mathrm{M}^{3+}=\mathrm{Al}^{3+}, \mathrm{Cr}^{3+}, \mathrm{Fe}^{3+}$, have important influence on multiple physiological and pathological processes. For example, high levels of $\mathrm{Al}^{3+}$ ions can give rise to degeneration of the cranial nerves and biological function damage, including cognitive deterioration, mental disorder and metabolic disturbance of calcium and phosphorus. ${ }^{3}$ Abnormal content of $\mathrm{Cr}^{3+}$ ions can trigger diabetes and cardiovascular diseases. ${ }^{4}$ The deficiency of $\mathrm{Fe}^{3+}$ ion, an essential trace element,

Key Laboratory of Cluster Science of Ministry of Education, Beijing Key Laboratory of Photoelectronic/Electrophotonic Conversion Materials, School of Chemistry and Chemical Engineering, Beijing Institute of Technology, Beijing, 100081, P. R. China. E-mail: zhijunge@bit.edu.cn, jinlwang@bit.edu.cn

$\dagger$ Electronic supplementary information (ESI) available: Characterization of NMR and MS spectra, UV-Vis absorption and fluorescence spectra, and crystallographic data. CCDC 1814395. For ESI and crystallographic data in CIF or other electronic format see DOI: $10.1039 / \mathrm{c} 8 \mathrm{qm} 00424 \mathrm{~b}$ can cause many physiological abnormalities such as hypoimmunity, decreased resistance to infection and anemia. ${ }^{5}$ Therefore, research on fluorescent materials for effectively detecting trivalent metal cations is of great significance.

To date, there are many reports with respect to fluorescent materials used for detecting metal ions; ${ }^{6}$ however, many of them are turn-off type sensors, resulting in output information inevitably suffering from background fluorescence interference and disadvantages in biosensing and bioimaging fields. ${ }^{7}$ Compared to turn-off sensors, turn-on sensors with excellent performance are more valuable, in particular for biological applications. ${ }^{8}$ Here, spirocyclic derivatives of Rhodamine B have been brought into focus because of their capability to switch between two structural forms by responding towards specific species; these two forms are fluorescence-off spirolactam form and fluorescence-on open-cycle amide form, and this has a close relationship with the decoration of molecular structure. ${ }^{9}$ The optimization of synthetic strategies, including the modification of amino groups of xanthene unit, carboxyphenyl ring and carboxylic acid group, enriched the structural composition of rhodamine dyes, which widened the application in specific fluorescence sensing field. ${ }^{10}$ For example, Prof. D. Das et al. reported a fluorescent sensor based on Rhodamine B and pyrene for detecting $\mathrm{Al}^{3+}$ in $2013 .{ }^{11}$ It was a turn-on type probe 
because it could transform the spirolactam form of Rhodamine B moiety into the amide form through chelation between the sensor and $\mathrm{Al}^{3+}$ ions. Moreover, considering the advantage in the convenience of recognition, fluorescence materials with sensitivity towards external stimuli provide a prospective platform to act as smart species for information security protection and data memory. ${ }^{12}$ Meanwhile, in terms of biosensors and labelling dyes, the superior emitting ability of materials in aggregated state is of great significance. However, the excellent photophysical property of many fluorescent sensors in aggregated state is weak in contrast to those in solution state due to aggregation caused quenching (ACQ), which originates from strong intermolecular $\pi-\pi$ interactions constructing non-radiation decay channel generally. ${ }^{13}$ As a promising solution, aggregation-induced emission (AIE) effect ${ }^{14}$ was reported by Prof. B. Z. Tang in 2001 and fluorescent materials based on AIE property have been attracting significant attention for sensors and solid-state luminescence. ${ }^{15}$ For instance, in 2016, Prof. A. Misra et al. reported aggregation-induced emission enhancement (AIEE) of a fluorescent sensor, pyrene-based Schiff base, for the detection of trace-level $\mathrm{Al}^{3+}$ with detection limit of $8.64 \mathrm{nM}$. The sensor exhibited around 200-fold fluorescence enhancement with the addition of $\mathrm{Al}^{3+}$ ions because of the chelation-enhanced fluorescence and AIEE effect. ${ }^{16}$ As a consequence, sensors with the capabilities of turn-on detection of metal cations and brilliant emission in an aggregated state for practical applications are worthy of further investigation. However, very few Rhodamine B derivative-based AIE fluorescent sensors have been reported for applications of turn-on detection of metal cations.

In this study, we designed and synthesized a novel AIE-active fluorescent sensor, TPEThRB, which is composed of a tetraphenylethene unit, spirocyclic Rhodamine B derivative, and an electron-rich thiophene ring. Tetraphenylethene fragment, as a traditional AIE fluorophore, can modulate TPEThRB molecule with AIE property and offer notable steric effect via its propellerlike configuration. The rhodamine segment acts as the indicator of trivalent metal cations via the structural change from looplocked spirolactam with no emission to open-loop amide with fluorescence emission. The thiophene unit as a conjugated bridge enriches the electron-donating ability and improves the interaction of TPEThRB molecule with metal ions.

Using turn-on fluorescence enhancement for trivalent metal cations and sensitive response towards $\mathrm{H}^{+}$ions with clear contrast of visible color and emission, the uses of TPEThRB for chemosensing, data protection and bioimaging are explored. Compared to conventional manner of metal ion chelation-induced ring-opening process of spirocyclic derivatives of Rhodamine $\mathrm{B},{ }^{17}$ trivalent metal cations $\left(\mathrm{Al}^{3+}, \mathrm{Cr}^{3+}\right.$ and $\left.\mathrm{Fe}^{3+}\right)$ with Lewis acid characteristics are specifically recognized by turn-on fluorescence and absorption analyses, which may be assigned to the synergy effect of protonated open-cycle and coordination interaction. Owing to the excellent luminescence properties in solid state and the response to $\mathrm{H}^{+}$ions with clear fluorescence and visible color changes, the circle of data writing, reading and erasing based on TPEThRB molecule can be achieved by means of trifluoroacetic acid vapor as the chromogenic reagent and diethylamine vapor as the eraser. Furthermore, in terms of effective emission in intracellular environment and the remarkable red shift of maximum fluorescent peak, the application of TPEThRB sensor in bioimaging of trivalent metal ions in HeLa cells was investigated. The results of ratio fluorescence imaging indicated that TPEThRB is suitable for fluorescent imaging of trivalent metal cations in living cells. The multifunctional AIEactive fluorescent materials have triggered intense research interest and the performances of TPEThRB were investigated thoroughly in this study.

\section{Results and discussion}

The target molecule TPEThRB was synthesized through facile Schiff-base condensation reaction of compounds $\mathbf{1}$ and 2, as shown in Scheme 1. The compounds $\mathbf{1}$ and $\mathbf{2}$ were obtained by relevant reference methods. ${ }^{18}$ The molecular structure was characterized by ${ }^{1} \mathrm{H}$ and ${ }^{13} \mathrm{C}$ NMR spectra, HR-MS spectra, a single crystal X-ray diffractometer, and theoretical calculation.

\section{AIE behavior and photophysical property}

To demonstrate the AIE characteristics of TPEThRB molecule, fluorescent spectra of $\mathrm{CH}_{3} \mathrm{CN}-\mathrm{H}_{2} \mathrm{O}$ mixture with different water fractions $\left(f_{\mathrm{w}}\right)$ were investigated. The dilute $\mathrm{CH}_{3} \mathrm{CN}$ solution of TPEThRB molecule exhibited weak blue fluorescence with maximum emission peak at $467 \mathrm{~nm}$ (Fig. 1a) and the emission spectra exhibited negligible variation when $f_{\mathrm{w}}$ in $\mathrm{CH}_{3} \mathrm{CN}-\mathrm{H}_{2} \mathrm{O}$ mixture was lower than $50 \%$. When $f_{\mathrm{w}}$ increased to $60 \%$, an intense blue-green fluorescence band with maximum emission peak at $492 \mathrm{~nm}$ appeared, and the maximum emission intensity of TPEThRB in $\mathrm{CH}_{3} \mathrm{CN}-\mathrm{H}_{2} \mathrm{O}$ mixture with $f_{\mathrm{w}}=70 \%$ was around 11-fold higher than that in pure $\mathrm{CH}_{3} \mathrm{CN}$ solution (Fig. 1b).

The AIE property was further verified by the fluorescence quantum yield $\left(\Phi_{\mathrm{F}}\right)$ and UV-Vis absorption spectra. The $\Phi_{\mathrm{F}}$ value of TPEThRB was 0.19 in dilute $\mathrm{CH}_{3} \mathrm{CN}$ solution and 6.24 in solid state, demonstrating clear enhancement in solid-state fluorescence quantum yield $\left(\alpha_{\mathrm{AIE}}=32.8\right)$. Furthermore, the UV-Vis absorption spectra of TPEThRB in $\mathrm{CH}_{3} \mathrm{CN}-\mathrm{H}_{2} \mathrm{O}$ mixtures with high water contents displayed Mie scattering effect, indicating the formation of nano-aggregates (Fig. S4, ESI $\dagger$ ). The sizes of TPEThRB in the $\mathrm{CH}_{3} \mathrm{CN}-\mathrm{H}_{2} \mathrm{O}$ mixtures with high water contents were investigated by dynamic light scattering (DLS). The DLS data demonstrated that the sizes of aggregates decrease with increasing volume fractions of water: 458, 447, 387, and $381 \mathrm{~nm}$ for $f_{\mathrm{w}}=60 \%, 70 \%, 80 \%$ and $90 \%$, respectively (Fig. S5, ESI $\dagger$ ); this is in agreement with the results of many other reported AIE systems. ${ }^{19}$ Herein, it is confirmed that TPEThRB exhibits AIE characteristics, which may play a great role in broadening the

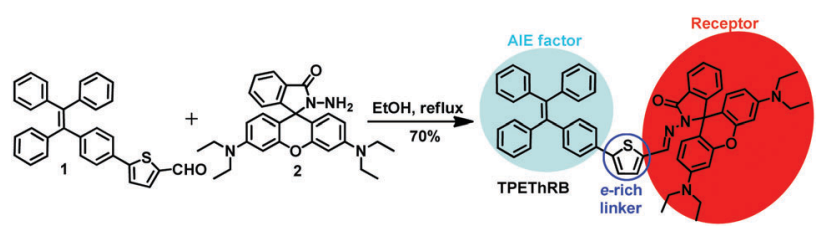

Scheme 1 Synthetic route of compound TPEThRB. 

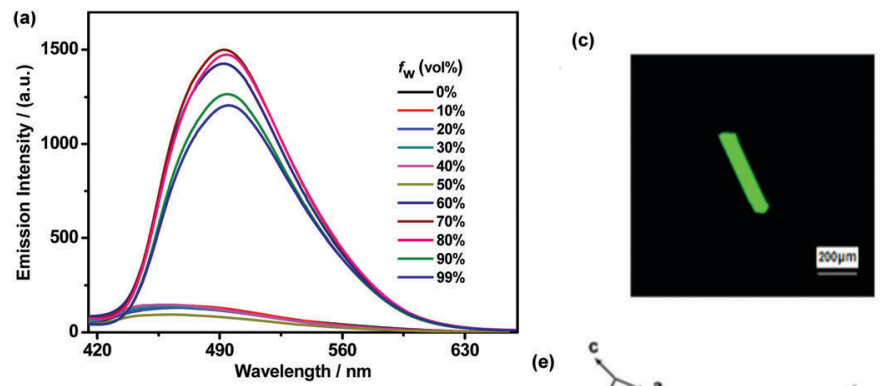

(d)
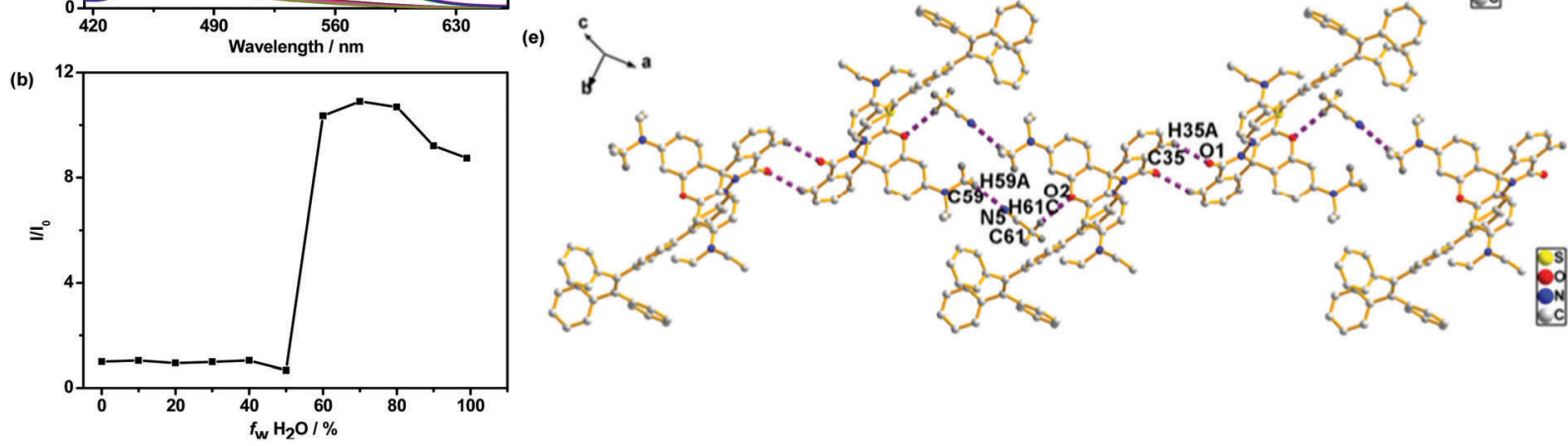

Fig. 1 (a) Fluorescence spectra of TPEThRB $(10 \mu \mathrm{M})$ in $\mathrm{CH}_{3} \mathrm{CN}-\mathrm{H}_{2} \mathrm{O}$ solution with different water fractions $\left(f_{\mathrm{w}}\right)$. (b) Plot of $\left(/ / I_{0}\right)$ vs. $f_{\mathrm{w}}$, where $I_{0}$ and $/$ are the emission intensities of TPEThRB in $\mathrm{CH}_{3} \mathrm{CN}$ and $\mathrm{CH}_{3} \mathrm{CN}-\mathrm{H}_{2} \mathrm{O}$ mixture. (c) Confocal laser scanning image. (d) Single-crystal structure of TPEThRB (CCDC 1814395) and (e) chain crystallography diagram. $\dagger$ TPEThRB single crystal was incubated in $\mathrm{CH}_{3} \mathrm{CN}$ solution and crystallographic data were collected at $296 \mathrm{~K}$.

application of TPEThRB in the fields of sensor, data protection and bioimaging.

Fortunately, a single crystal of TPEThRB was obtained, which was assigned to the triclinic crystal system with a space group of $P \overline{1}$. The fluorescence image from confocal laser scanning microscope showed that the crystal emitted green fluorescence and had a column-like appearance (Fig. 1c). Bright fluorescence in aggregated state and the highly twisted molecule conformation (Fig. 1d) are consistent with AIE property. In addition, ordered molecule arrangement and antiparallel packing with thiophene ring were confirmed by X-ray single-crystal analysis (Fig. S6, ESI†). The regular orientation and alignment of the single crystal of TPEThRB are derived from multiple interactions containing hydrogen bond interactions and $\pi-\pi$ stacking. For example, the 1D chain structure (Fig. 1e) was constructed by $\mathrm{C}-\mathrm{H} \cdots \mathrm{N}$ $\left[\mathrm{C} 59-\mathrm{H} 59 \mathrm{~A} \cdots \mathrm{N} 5, \quad 2.53 \AA, \quad 3.40(1) \AA, 1^{\circ}\right]$ and $\mathrm{C}-\mathrm{H} \cdots \mathrm{O}$

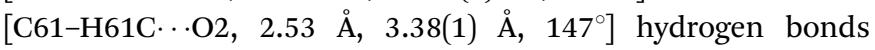
between the host molecules and non-bonding $\mathrm{CH}_{3} \mathrm{CN}$ and $\mathrm{C}-\mathrm{H} \cdots \mathrm{O}\left[\mathrm{C} 35-\mathrm{H} 35 \mathrm{~A} \cdots \mathrm{O} 1,2.55 \AA\right.$, 3.31(1) $\AA$, 139 ${ }^{\circ}$ hydrogen bonds between adjacent host molecules. Moreover, the $\pi-\pi$ stacking interaction between thiophene rings with a distance of 3.943(2) A could contribute to the formation of the 2D layer structure. The $\mathrm{C}-\mathrm{H} \cdots \pi$ interaction with a distance of 3.634 (6) $\AA$ between the thiophene ring and the ethyl group was also observed. The ordered single-crystal construction of TPEThRB with column-like appearance may offer the possibility to extend applications in optical devices.

\section{Optical response of TPEThRB probe towards ions}

To check the performance of TPEThRB as a fluorescent sensor, fluorescence spectra of TPEThRB with trivalent metal cations, $\mathrm{M}^{3+}=\mathrm{Al}^{3+}, \mathrm{Cr}^{3+}, \mathrm{Fe}^{3+}$, and $\mathrm{H}^{+}$ion in $\mathrm{CH}_{3} \mathrm{CN}-\mathrm{H}_{2} \mathrm{O}$ mixture
$(3: 2, v / v)$ were investigated. As shown in Fig. 2, with the addition of trivalent metal cations and $\mathrm{H}^{+}$ions, similar maximum emissions at $588 \mathrm{~nm}$ for trivalent metal cations and at $582 \mathrm{~nm}$ for $\mathrm{H}^{+}$ion were observed and the mixtures presented visible color change from colorless to pink. The interaction between the sensor and the four species could reach the terminal state within six minutes at the most (Fig. S7, ESI $\dagger$ ), accompanied with turn-on maximum fluorescence intensities (60-fold for $\mathrm{H}^{+}$, 29-fold for $\mathrm{Al}^{3+}, 28$-fold for $\mathrm{Cr}^{3+}$ and 17-fold for $\mathrm{Fe}^{3+}$ ), and the fluorescence quantum yields of TPEThRB in the absence and presence of metal ions were also measured: 0.17 for blank TPEThRB solution, 1.20 for $\mathrm{H}^{+}$ion, 0.67 for $\mathrm{Al}^{3+}$ ion, 0.51 for $\mathrm{Cr}^{3+}$ ion and 0.27 for $\mathrm{Fe}^{3+}$ ion. Furthermore, on the basis of Job's plot (Fig. S8, ESI $\dagger$ ) of $\left[I^{\prime} / I_{0}{ }^{\prime}-1\right]$ versus the concentration of trivalent metal cations and $\mathrm{H}^{+}$, the estimated detection limits (LOD) by $3 \sigma / s$ method were $3.2 \mu \mathrm{M}$ for $\mathrm{Fe}^{3+}, 4.8 \mu \mathrm{M}$ for $\mathrm{Al}^{3+}, 11.9 \mu \mathrm{M}$ for $\mathrm{Cr}^{3+}$ and $0.93 \mu \mathrm{M}$ for $\mathrm{H}^{+}$(Fig. S9, ESI ${ }^{\dagger}$ ); here, $I^{\prime}$ and $I_{0}{ }^{\prime}$ were assigned to the maximum emission intensities at $588 \mathrm{~nm}$ and at $582 \mathrm{~nm}$ in the presence and absence of trivalent metal cations and $\mathrm{H}^{+}$ion, $\sigma$ is the standard deviation of blank measurements, and $s$ is the slope of the Job's plot. The data of LOD are at moderate level compared with that for reported sensors for trivalent metal ions (Table S1, $\mathrm{ESI} \dagger)^{20}{ }^{20}$ The evident fluorescence changes in the long wavelength region displayed the sensitive response of TPEThRB to the four species. In addition to more remarkable enhancement of maximum emission at $582 \mathrm{~nm}$, the $\mathrm{H}^{+}$ion has a relatively large influence on the maximum emission in the short wavelength region of TPEThRB than trivalent metal cations. The presence of $\mathrm{H}^{+}$ion contributed to the structural transformation of the loop-locked spirolactam into an open-loop amide, resulting in extended conjugation length and improvement in intramolecular charge transfer. Accordingly, there was a moderate red shift of 

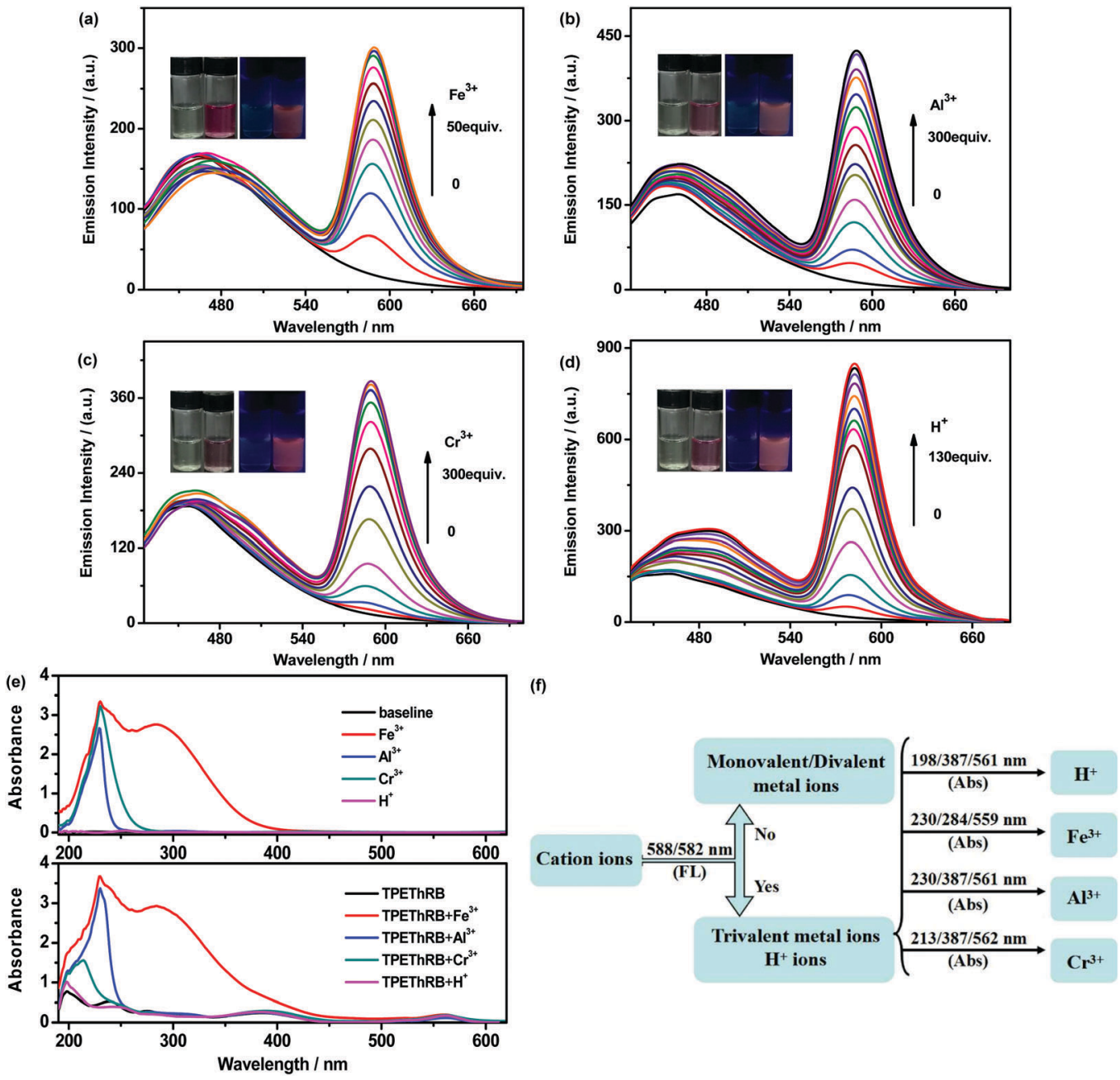

Fig. 2 Fluorescence titration spectra of compound TPEThRB $(10 \mu \mathrm{M})$ in $\mathrm{CH}_{3} \mathrm{CN}-\mathrm{H}_{2} \mathrm{O}$ solution with different species (a) $\mathrm{Fe}^{3+}$, (b) $\mathrm{Al}^{3+}$, (c) $\mathrm{Cr}^{3+}$, (d) $\mathrm{H}^{+}$. Inset: The photographs of TPEThRB in $\mathrm{CH}_{3} \mathrm{CN}-\mathrm{H}_{2} \mathrm{O}$ solution with $\mathrm{Fe}^{3+}, \mathrm{Al}^{3+}, \mathrm{Cr}^{3+}$ and $\mathrm{H}^{+}$under natural light (left) and UV lamp illumination (right). (e) UV-Vis absorption spectra of compound TPEThRB $(10 \mu \mathrm{M})$ with four species, $\mathrm{H}^{+}, \mathrm{Al}^{3+}, \mathrm{Cr}^{3+}$ and $\mathrm{Fe}^{3+}$ in $\mathrm{CH}_{3} \mathrm{CN}_{-} \mathrm{H}_{2} \mathrm{O}$ solution and only $\mathrm{H}^{+}, \mathrm{Al}^{3+}, \mathrm{Cr}^{3+}$ and $\mathrm{Fe}^{3+}$ in $\mathrm{CH}_{3} \mathrm{CN}-\mathrm{H}_{2} \mathrm{O}$ solution. (f) Process scheme to recognize $\mathrm{H}^{+}, \mathrm{Al}^{3+}, \mathrm{Cr}^{3+}, \mathrm{Fe}^{3+}$ by using fluorescence and UV-Vis absorption spectra.

the maximum emission peak from 460 to $490 \mathrm{~nm}$ with slight increase in fluorescence intensity. In contrast, the presence of trivalent metal ions, $\mathrm{Fe}^{3+}, \mathrm{Al}^{3+}$ and $\mathrm{Cr}^{3+}$, had negligible effect on the emission in short wavelength region of TPEThRB due to coordination interaction. Moreover, to evaluate the reversibility of TPEThRB responding to trivalent metal ions, fluorescence spectra with the addition of disodium salt of EDTA (EDTA-2Na) were investigated. Compared to notable fluorescence enhancement of maximum emission peaks at $588 \mathrm{~nm}$ in the presence of trivalent metal ions, the addition of EDTA-2Na (5 equiv.) into TPEThRB-M ${ }^{3+}$ complexes resulted in $6 \mathrm{~nm}$ blue shift of maximum characteristic emission peak from 588 to $582 \mathrm{~nm}$ accompanied with sharp decrease in emission intensity (Fig. 3a). The results indicated that the coordination interaction between TPEThRB and trivalent metal ions was removed owing to better coordination affinity between EDTA-2Na and trivalent metal ions, but the effect of acidic hydrolysis on TPEThRB was still observed, which is in agreement with the proposed response mechanism of the synergy of proton-induced open-cycle and the following coordination interaction.
To obtain better insights into the specific response capability of TPEThRB sensor towards trivalent metal cations and $\mathrm{H}^{+}$ions, UV-Vis absorption spectra in $\mathrm{CH}_{3} \mathrm{CN}-\mathrm{H}_{2} \mathrm{O}$ mixture $(3: 2, \mathrm{v} / \mathrm{v})$ were explored. Distinct characteristic absorption peaks appeared in the presence of trivalent metal ions and $\mathrm{H}^{+}$ion. Based on selective matching with characteristic absorption peaks together with fluorescence spectra, it is possible to further distinguish the four ions from each other. The appearance of maximum emission peak at 588 and $582 \mathrm{~nm}$ is adopted to divide trivalent metal ions and $\mathrm{H}^{+}$ion from others. In view of the intrinsic maximum absorption at 230 and $284 \mathrm{~nm}$ for $\mathrm{Fe}^{3+}$ as well as that at $230 \mathrm{~nm}$ for $\mathrm{Al}^{3+}$ and $\mathrm{Cr}^{3+}$ in $\mathrm{CH}_{3} \mathrm{CN}-\mathrm{H}_{2} \mathrm{O}$ mixture, maximum absorption peaks at 559, 284 and $230 \mathrm{~nm}$ could be used to identify $\mathrm{Fe}^{3+}$ ion, maximum absorption peaks at 561, 387 and $230 \mathrm{~nm}$ may distinguish $\mathrm{Al}^{3+}$ ions, and maximum absorption peaks at 562, 387 and $213 \mathrm{~nm}$ could verify $\mathrm{Cr}^{3+}$ ion. In addition, the maximum absorption peaks at 561, 387 and $198 \mathrm{~nm}$ were assigned to $\mathrm{H}^{+}$ ion. Therefore, with the combination of the analyses of fluorescence and UV-Vis absorption spectra, we infer that the TPEThRB 

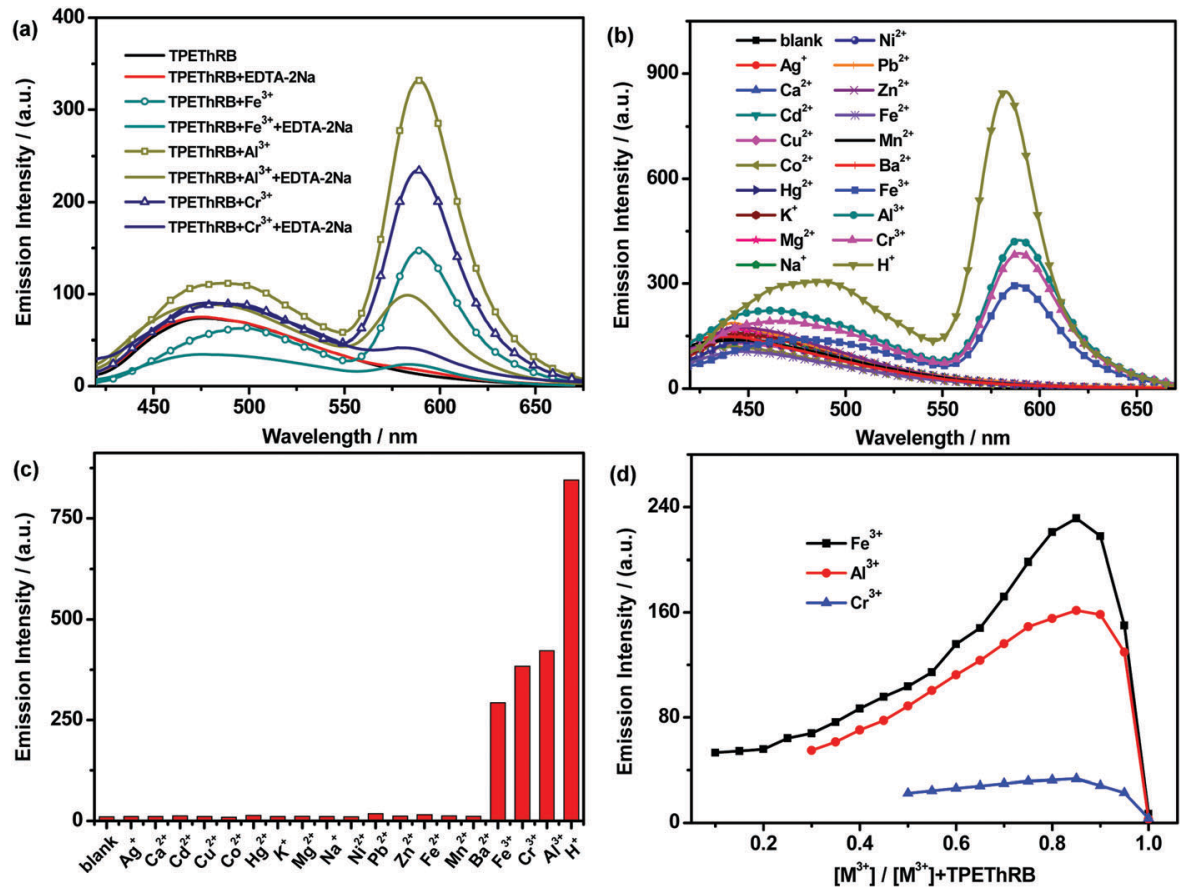

Fig. 3 (a) Fluorescence spectra of TPEThRB by addition of trivalent metal ions and EDTA-2Na. (b) UV-Vis absorption spectra of TPEThRB with monovalent and divalent metal ions. (c) Histogram of fluorescence emission of compound TPEThRB (10 $\mu$ M) in $\mathrm{CH}_{3} \mathrm{CN}-\mathrm{H}_{2} \mathrm{O}$ solution with different valence cations and $(d)$ the Job's plot of $\left[\mathrm{M}^{3+}\right] /\left[\mathrm{M}^{3+}\right]+[$ TPEThRB $]$ vs. emission intensity with a total concentration of $\left[\right.$ TPEThRB] $+\left[\mathrm{M}^{3+}\right]=90 \mu \mathrm{M}$; $\mathrm{M}^{3+}=\mathrm{Fe}^{3+}, \mathrm{Al}^{3+}$ and $\mathrm{Cr}^{3+}$.

sensor can sensitively and individually recognize trivalent metal ions and $\mathrm{H}^{+}$ions.

\section{Selectivity}

It is well-known that there are two forms for Rhodamine B derivatives: loop-locked spirolactam with no emission and open-loop amide with fluorescence emission. ${ }^{21}$ Upon responding towards analytes with structural transformation from the former type into the latter one, the characteristic absorption peak and characteristic fluorescence peak in the long wavelength range will be observed. Owing to the requirement of specifically detecting target species for sensors, the fluorescence and UV-Vis absorption spectra of TPEThRB interacting with various ions were investigated, and these ions include $\mathrm{Ag}^{+}, \mathrm{K}^{+}, \mathrm{Na}^{+}, \mathrm{Ca}^{2+}, \mathrm{Cd}^{2+}, \mathrm{Cu}^{2+}, \mathrm{Co}^{2+}$, $\mathrm{Hg}^{2+}, \mathrm{Mg}^{2+}, \mathrm{Ni}^{2+}, \mathrm{Pb}^{2+}, \mathrm{Zn}^{2+}, \mathrm{Fe}^{2+}, \mathrm{Mn}^{2+}, \mathrm{Ba}^{2+}, \mathrm{Fe}^{3+}, \mathrm{Al}^{3+}, \mathrm{Cr}^{3+}$ and $\mathrm{H}^{+}$. The results displayed that characteristic maximum emission peak (Fig. 3b) and characteristic maximum absorption peak (Fig. 2e and Fig. S10, ESI $\dagger$ ) in the long wavelength region were only noticeably observed for the mixture of TPEThRB molecule with trivalent metal cations and $\mathrm{H}^{+}$ion. Compared to the trivalent metal ions and $\mathrm{H}^{+}$ion giving rise to open-loop response, the tested monovalent and divalent metal ions exerted no impact on the structural change of spirocyclic Rhodamine B subunit and the molecule still retained the primary loop-locked spirolactam form, due to which emission spectra and UV-Vis absorption spectra remained unchanged in the long wavelength region. Thus, TPEThRB sensor exhibits excellent selectivity concurrently with fine response capability (Fig. 3c), which is in favor of application in bioimaging.
To clarify the specific recognition of trivalent metal cations from monovalent and divalent metal cations, the response mechanism was further studied. The Job's plots of the emission intensity versus the molar fraction of $\mathrm{M}^{3+}$ in the mixture of $\mathrm{M}^{3+}$ and TPEThRB with total concentration of $90 \mu \mathrm{M}$ exhibited the same maximum molar fraction at 0.85 (Fig. 3d), which illustrated approximate 1:6 ratio for the TPEThRB- $\mathbf{M}^{3+}$ complex. The abnormal stoichiometry indicated that the ring-opening is not solely induced by the coordination interaction with metal ions. Considering the steric effect modulated by propeller-like conformation of TPE moiety, compact structural architecture and nonresponse towards monovalent/divalent metal ions, the interaction affinity of TPEThRB towards metal ions may not be strong enough to trigger the conventional metal ion chelationinduced ring-opening of spirocyclic Rhodamine B subunit. ${ }^{22}$ With the assistance of acidic condition produced by the hydrolysis of trivalent metal cations, Lewis acid characteristics of $\mathrm{Al}^{3+}$, $\mathrm{Cr}^{3+}$ and $\mathrm{Fe}^{3+}$ may play a great role in the interaction of TPEThRB sensor with trivalent metal ions. As for the ability of generating protons through acidic hydrolysis, it is well known that $\mathrm{Fe}^{3+}$ is the most capable, followed by $\mathrm{Al}^{3+}$ and $\mathrm{Cr}^{3+}$ in that order. ${ }^{23}$ The order is in agreement with the estimated detection limit of TPEThRB sensor for the three trivalent metal ions. With regard to the fluorescence spectra of TPEThRB with trivalent metal ions in $\mathrm{CH}_{3} \mathrm{CN}-\mathrm{PBS}$ mixture $(10 \mathrm{mM}, \mathrm{pH}=7.4)$, there was no obvious fluorescent response until the concentrations of trivalent metal ions were at a relatively high level (Fig. S11, ESI $\dagger$ ), indicating that acidic condition is important to trigger the response. Meanwhile, compared to the ${ }^{1} \mathrm{H}$ NMR spectra of blank TPEThRB sample, the 


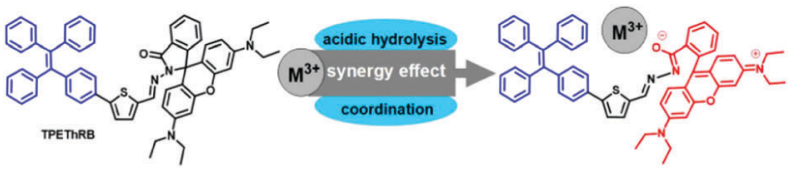

Scheme 2 The proposed response mechanism of TPEThRB towards $\mathrm{M}^{3+}$.

$-\mathrm{CH}=\mathrm{N}-$ proton displayed an evident downfield shift from 8.83 to $9.46 \mathrm{ppm}$ with the addition of $\mathrm{H}^{+}$ions. However, the characteristic-CHO- proton (9.86 ppm) of compound 1 was not observed (Fig. S12, ESI $\dagger$ ), implying that the acidic condition results in spirolactam ring-opening rather than the hydrolysis of Schiff base because compound 1 was not regenerated. The above results further indicate that the interaction between the trivalent metal ions and TPEThRB can be ascribed to the synergy of protonated open-cycle and coordination interaction rather than the conventional mechanism of metal chelation reaction alone (Scheme 2).

Besides the appearance of characteristic absorption peak and fluorescence peak, the transformation to an open-loop amide form is conducive to alleviate the packed configuration and modulate electron distribution. The extended spatial construction of the molecule and the electron-enriched amide in combination with the conjugated thiophene linker can improve the coordination affinity between TPEThRB sensor and trivalent metal ions. The coordination interactions with the trivalent metal ions have significant influence on their UV-Vis absorption spectra, contributing to further recognize the responsive four species distinctly. Additionally, the ${ }^{1} \mathrm{H}$ NMR spectra of TPEThRB were recorded with trivalent metal ions to further clarify the interaction between probe and metal ions (Fig. 4). The influence of the trivalent metal ions on chemical shifts was clear. In particular, the signal peak of $-\mathrm{CH}=\mathrm{N}-$ proton broadened and shifted upfield to different degrees, which is consistent with a previous report that demonstrated the ring-opening of Rhodamine spirolactam and coordination interaction. ${ }^{24}$
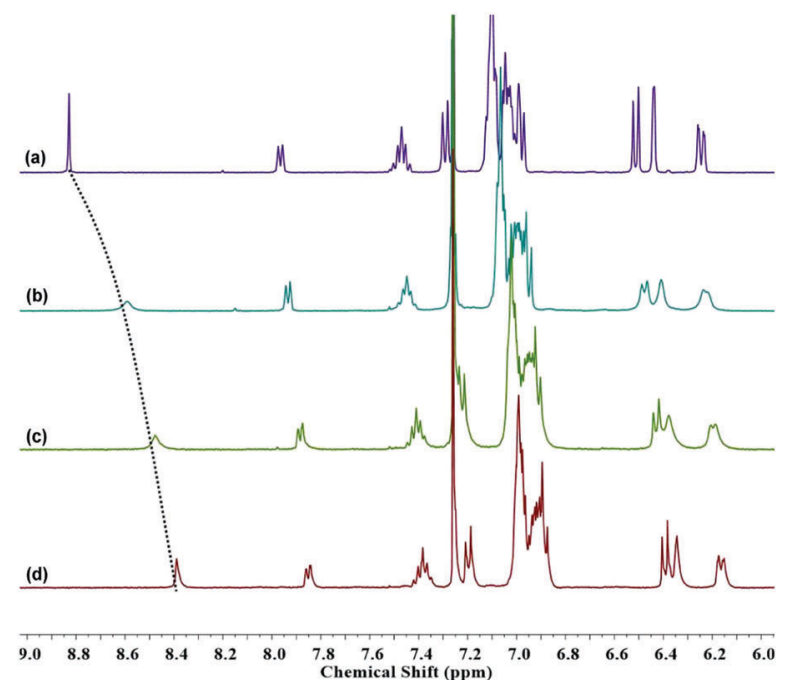

Fig. $4{ }^{1} \mathrm{H}$ NMR spectra (in $\mathrm{CDCl}_{3}-\mathrm{CD}_{3} \mathrm{OD}$ ) of TPEThRB in the (a) absence and presence of (b) $\mathrm{Fe}^{3+}$, (c) $\mathrm{Al}^{3+}$ and (d) $\mathrm{Cr}^{3+}$ ions.

\section{Theoretical calculation}

To maintain the optimized conformation, appropriate spatial arrangement can diminish the structural tension originating from the propeller-like configuration of TPE unit and compact architecture connection. To better understand the steric effect on the molecule configuration and response mechanism, DFT calculations with the B3LYP method have been performed (Fig. S13, ESI $\dagger$ ). The calculated results of spatial configuration showed that the tricyclic subunit of spirocyclic Rhodamine $\mathrm{B}$ moiety was almost perpendicular to bicyclic subunit. The result is in agreement with that of single crystal analysis. The orbital electron distribution showed that the highest occupied molecular orbital (HOMO) is mainly located in tetraphenylethene and conjugated bridge, and the lowest unoccupied molecular orbital (LUMO) is distributed on partial tetraphenylethene, conjugated bridge and bicyclic segment of the spirocyclic Rhodamine B moiety. Due to almost perpendicular configuration, the tricyclic subunit of spirocyclic Rhodamine B moiety has no effective contribution towards orbital distribution, which largely impedes the extension of electron delocalization. Moreover, it is the lone pair electrons of nitrogen atom situated in tricyclic subunit that is the starting point to take part in electron rearrangement. The perpendicular configuration and steric hindrance inevitably inhibit triggering of metal ion chelation-induced ring-opening, resulting in no response to the monovalent and divalent metal ions. In contrast, the open-loop form induced by the acidic hydrolysis of trivalent metal ions effectively decreases the steric effect and increases the conjugation length, which is in favour of the interaction between TPEThRB sensor and trivalent metal ions.

\section{Data protection}

Materials with photophysical property of long emission lifetime and multi-stimuli response property, such as temperature, solvent, illumination, mechanical force and vapor, for data recording, encryption and decryption are of great value in the fields of information recording and security technologies. ${ }^{25}$ As for luminogens, which are sensitive to acid vapor, it is interesting to exploit their acidochromic property with colorimetric and fluorescent variations for practical applications. ${ }^{26}$

The AIE-active TPEThRB can respond to $\mathrm{H}^{+}$with evident fluorescence and color changes; thus, a promising strategy to realize data recording, encryption and decryption by adopting TPEThRB as the smart material has been actualized. A strip of filter paper as a substrate was immersed in tetrahydrofuran (THF) solution of TPEThRB for a few seconds and then, we took it out and dried it by volatilizing to make TPEThRB deposit on the filter paper strip. The filter paper strip loaded with TPEThRB was covered by a template with engraving hollowed words 'AIE' and fumigated with trifluoroacetic acid (TFA) vapor for one minute. After removing the hollowed template, amaranthine pattern 'AIE' appeared on the filter paper strip together with clear contrast of fluorescent change between the blue-green emission of substrate and red-orange emission of the words 'AIE' (Fig. 5a and b). Moreover, the 'AIE' pattern on the strip of filter paper disappeared thoroughly after being exposed to diethylamine 

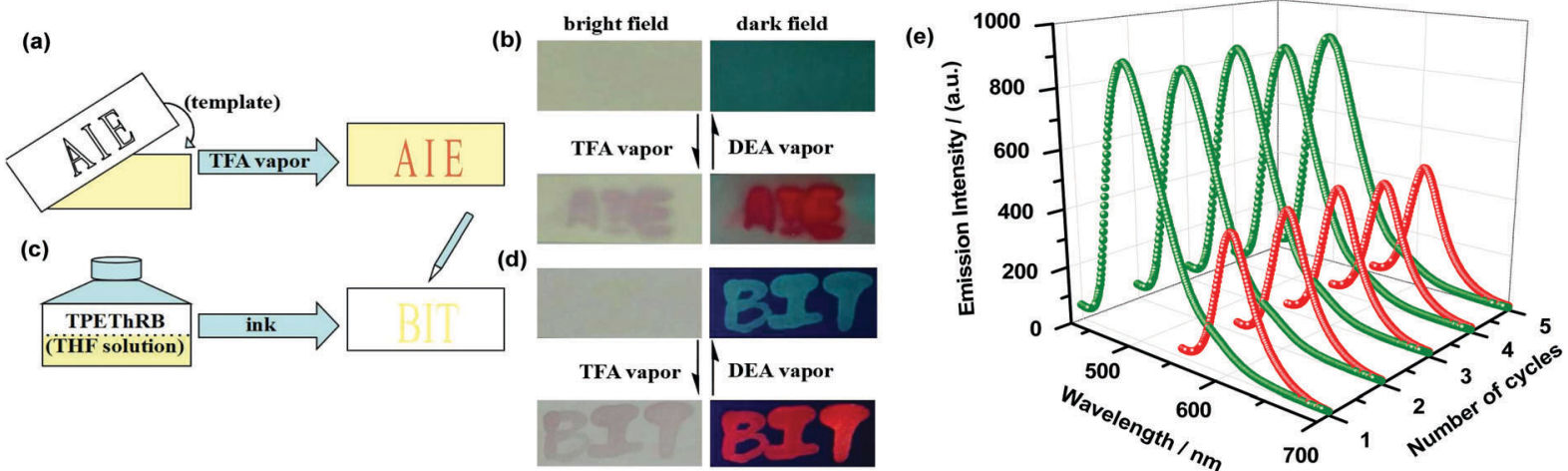

Fig. 5 (a) Schematic diagrams and (b) photographs of data protection based on TPEThRB in template manner under natural light (up) and UV lamp (down); (c) diagram and (d) corresponding photograph of ink writing; (e) fluorescence spectra of TPEThRB exposed to TFA vapor and DEA vapor and reversible cycles.

(DEA) vapor for a few seconds. The above processes represent the data writing and erasing process.

Similarly, the THF solution of TPEThRB could also be used as a kind of encrypted ink to write words 'BIT' on a blank filter paper. The faint yellow words 'BIT' are illegible under natural light (Fig. 5c and d), whereas words with intensive blue-green fluorescent emission were clearly observed under UV lamp illumination owing to the outstanding AIE feature of the TPEThRB molecule, where the background blue luminescence originated from the blank filter paper margin under $365 \mathrm{~nm}$ UV light irradiation (Fig. S14, ESI $\dagger$ ). After exposure to TFA vapor for one minute, the filter paper strip distinctly presented amaranthine words 'BIT' with orange-red emission; then, after fumigation with DEA vapor for a few seconds, the words 'BIT' on the strip of filter paper returned to the original state. The above results indicated that TFA vapor acted as a chromogenic reagent to make information emerge clearly, and DEA vapor acted as an eraser to clean out the information conversely, which could be repeated many times (Fig. 5e). Due to the performances of writing, reading and erasing based on the stimuli-responsive AIE molecules, they can serve as promising candidates for the research of data recording and information security protection.

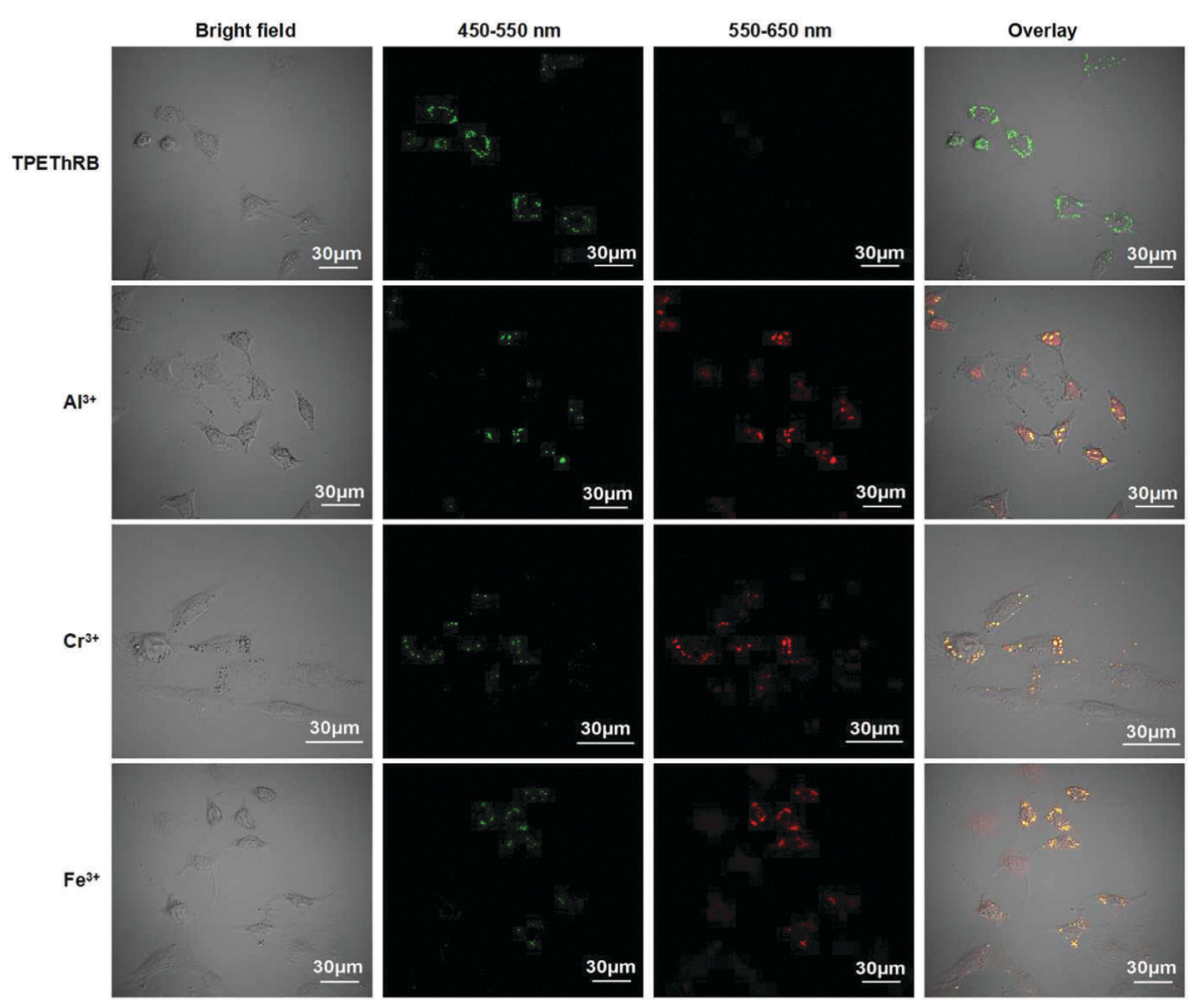

Fig. 6 The confocal laser scanning images of HeLa cells incubated with TPEThRB $(10 \mu \mathrm{M})$ and $\mathrm{Al}^{3+}(50 \mu \mathrm{M}), \mathrm{Cr}^{3+}(50 \mu \mathrm{M})$ and Fe ${ }^{3+}(25 \mu \mathrm{M})$ including bright-field images, fluorescence (450-550 nm channel and 550-650 nm channel) images and overlay images. 


\section{Bioimaging}

Materials with AIE characteristics are advantageous for applications in intracellular environment. ${ }^{27}$ Owing to the remarkable fluorescent responses of TPEThRB molecule towards the trivalent metal ions in $\mathrm{CH}_{3} \mathrm{CN}-\mathrm{H}_{2} \mathrm{O}$ solution (Fig. S15, ESI $\dagger$ ), the ability of TPEThRB sensor for the detection of trivalent metal cations $\left(\mathrm{M}^{3+}=\mathrm{Al}^{3+}, \mathrm{Cr}^{3+}, \mathrm{Fe}^{3+}\right)$ in HeLa cells was investigated by confocal fluorescence microscopy (Fig. 6). The green fluorescence channel of $450-550 \mathrm{~nm}$ and red fluorescence channel of $550-650 \mathrm{~nm}$ were selected to illustrate the performance of TPEThRB in bioimaging. The images showed that for the blank contrast group, the green fluorescence channel was bright and red fluorescence channel was dark when HeLa cells were incubated with TPEThRB sensor $(10 \mu \mathrm{M})$ for $30 \mathrm{~min}$ at $37^{\circ} \mathrm{C}$. After trivalent metal ions, i.e., $\mathrm{Al}^{3+}(50 \mu \mathrm{M}), \mathrm{Cr}^{3+}(50 \mu \mathrm{M})$ and $\mathrm{Fe}^{3+}(25 \mu \mathrm{M})$ ions were added in the three blank culture media for incubation for another $30 \mathrm{~min}$, both fluorescence channels of three test groups were visibly lightened. The ratio images of test groups having trivalent metal cations presented a striking contrast to the single channel images of the control group. The results indicated that TPEThRB sensor was capable of responding to trivalent metal ions in living cells, which is consistent with its performance in vitro. The AIE characteristics make the TPEThRB molecule retain its fine photophysical property and ratio fluorescence response ability towards trivalent metal cations within intracellular surroundings. Therefore, the AIE-active TPEThRB molecule can be a promising sensor for bioimaging of trivalent metal ions in HeLa cells.

\section{Conclusion}

In summary, we have rationally designed and synthesized a turnon AIE-active sensor, TPEThRB, containing tetraphenylethene with AIE characteristics, Rhodamine spirolactam as the receptor of target analytes and thiophene ring as the electron-rich conjugated linker. TPEThRB sensor can exhibit sensitive and specific turn-on response to trivalent metal cations with visible color change owing to the structural change of spirocyclic Rhodamine B moiety from a loop-locked spirolactam form to an open-loop amide form. Based on the abnormal stoichiometry of the interaction between TPEThRB and trivalent metal cations, we propose that the effective response may be ascribed to the synergy of protonated open-cycle and coordination interaction because of the steric effect caused by the propeller-like conformation of TPE unit and compact conjugated connection. A comprehensive investigation of analyses of acidic hydrolysis capability, efficient fluorescent response towards $\mathrm{H}^{+}$ions, response delay towards trivalent metal cations in $\mathrm{CH}_{3} \mathrm{CN}-$ PBS mixture and theoretical calculation can contribute to verify this assumption. In view of excellent acidochromism property with outstanding AIE feature, the utility of TPEThRB molecule for reversible data protection was realized on the substrate of filter paper; TFA vapor and DEA vapor acted as the chromogenic reagent and eraser, respectively. Furthermore, based on the advantage of AIE characteristics and the remarkable $128 \mathrm{~nm}$ red-shift of maximum emission in the presence of trivalent metal ions, the collected red fluorescence channel of bioimaging in HeLa cells was lightened by the addition of trivalent metal cations.
Hence it is demonstrated that TPEThRB is a viable chemosensor for trivalent metal cations in living cells. Our results indicate that AIE-active Rhodamine B-based materials will be promising candidates for multifunctional practical applications in fluorescence sensors, information protection and bioimaging fields.

\section{Experimental section}

${ }^{1} \mathrm{H}$ and ${ }^{13} \mathrm{C}$ NMR spectra were recorded on a Bruker ARX-400 (400 MHz) spectrometer in $\mathrm{CDCl}_{3}$ as reference using tetramethylsilane (TMS; $\delta=0$ ) as an internal standard. ESI MS was performed on an AGILENT Q-TOF 6520 liquid chromatograph mass spectrometer. Single crystal XRD was measured on an Agilent Gemini Ultra X-ray diffractometer. Fluorescence spectra and UV-Vis absorption spectra were obtained using F-7000 fluorescence spectrophotometer and UH5300 UV-Vis spectrophotometer, respectively. The theoretical calculation was performed using the Gaussian 09 suite of programs. The optimizations of the structures were performed by using B3LYP DFT. Fluorescence images of HeLa cells and single crystal of TPEThRB molecule were measured with an Olympus FluoView FV 1000 laser scanning confocal microscope.

\section{Synthesis of compound TPEThRB}

Compound 1 ( $0.13 \mathrm{~g}, 0.29 \mathrm{mmol})$ was added into $20 \mathrm{~mL}$ absolute ethanol solution of compound $2(0.11 \mathrm{~g}, 0.25 \mathrm{mmol})$ and then, the mixture was refluxed for $8 \mathrm{~h}$. After cooling to room temperature, ethanol was removed and the crude product was purified by silica gel column chromatography eluting with dichloromethane: petroleum ether $(2: 1)$ to obtain the product as a yellow solid $(0.18 \mathrm{~g}, 70 \%) .{ }^{1} \mathrm{H}$ NMR $\left(400 \mathrm{MHz}, \mathrm{CDCl}_{3}\right)$ : $\delta(\mathrm{ppm}), 8.83(\mathrm{~s}, 1 \mathrm{H},-\mathrm{N}=\mathrm{CH}-), 7.94-7.99(\mathrm{~d}, 1 \mathrm{H}, J=7.2 \mathrm{~Hz}$, Ar-H), 7.43-7.51 (m, 2H, Ar-H), 7.27-7.31 (d, $2 \mathrm{H}, J=8.0 \mathrm{~Hz}$, $\mathrm{Ar}-\mathrm{H})$, 7.07-7.14 (m, 10H, Ar-H), 6.96-7.06 (m, 10H, Ar-H), 6.49-6.54 (d, $2 \mathrm{H}, J=8.8 \mathrm{~Hz}, \mathrm{Ar}-\mathrm{H}), 6.42-6.46(\mathrm{~d}, 2 \mathrm{H}, J=2.0 \mathrm{~Hz}$, Ar-H), $6.22-6.28(\mathrm{dd}, 2 \mathrm{H}, J=8.8 \mathrm{~Hz}, 2.0 \mathrm{~Hz}, \mathrm{Ar}-\mathrm{H}), 3.27-3.37$ (q, $8 \mathrm{H}, J=7.2 \mathrm{~Hz}, \mathrm{CH}_{2}$ ), $1.13-1.18\left(\mathrm{t}, 12 \mathrm{H}, J=7.2 \mathrm{~Hz}, \mathrm{CH}_{3}\right.$ ). ${ }^{13} \mathrm{C}$ NMR (100 MHz, $\left.\mathrm{CDCl}_{3}\right): \delta(\mathrm{ppm}), 164.7,153.3,151.5,148.9$, 146.4, 143.7, 143.6, 143.50, 143.48, 142.2, 141.3, 140.3, 140.2, $133.2,132.0,131.8,131.4,131.34,131.31,130.6,129.5,128.3$, $128.0,127.8,127.7,127.6,126.6,126.55,126.48,125.0,123.8$, 123.3, 122.8, 108.0, 106.1, 98.0, 66.2, 44.3, 12.6. HR-MS (ESI) $(\mathrm{m} / \mathrm{z})$ : calculated for $\mathrm{C}_{59} \mathrm{H}_{52} \mathrm{~N}_{4} \mathrm{O}_{2} \mathrm{~S}:$ 881.3889, found: $881.3888\left(\mathrm{M}+\mathrm{H}^{+}\right)$.

\section{Conflicts of interest}

There are no conflicts to declare.

\section{Acknowledgements}

This work was financially supported by grants from the Natural Science Foundation of China (No. 21672023, 21472012, 21374010); J.-L. W. was supported by the Thousand Youth Talents Plan of China and Beijing Natural Science Foundation (2152027). The authors thank Prof. Yuping Dong (Beijing Institute of Technology) 
for fruitful discussion. The authors thank Analysis \& Testing Center, Beijing Institute of Technology for NMR and TGA experiments.

\section{References}

1 (a) T. Shan, Y. Liu, X. Tang, Q. Bai, Y. Gao, Z. Gao, J. Li, J. Deng, B. Yang, P. Lu and Y. Ma, ACS Appl. Mater. Interfaces, 2016, 8, 28771-28779; (b) J. Yang, L. Li, Y. Yu, Z. Ren, Q. Peng, S. Ye, Q. Li and Z. Li, Mater. Chem. Front., 2017, 1, 91-99; (c) J. Yang, J. Huang, Q. Li and Z. Li, J. Mater. Chem. C, 2016, 4, 2663-2684; (d) H. Luo, S. Chen, Z. Liu, C. Zhang, Z. Cai, X. Chen, G. Zhang, Y. Zhao, S. Decurtins, S.-X. Liu and D. Zhang, Adv. Funct. Mater., 2014, 24, 4250-4258; (e) Z. Li, X. Yan, F. Huang, H. Sepehrpour and P. J. Stang, Org. Lett., 2017, 19, 5728-5731; $(f)$ J. J. Gruskos, G. Zhang and D. Buccella, J. Am. Chem. Soc., 2016, 138, 14639-14649; ( $g$ ) J. Li, Y. Chen, T. Chen, J. Qiang, Z. Zhang, T. Wei, W. Zhang, F. Wang and X. Chen, Sens. Actuators, B, 2018, 268, 446-455; (h) D. Wang, H. Su, R. T. K. Kwok, G. Shan, A. C. S. Leung, M. M. S. Lee, H. H. Y. Sung, I. D. Williams, J. W. Y. Lam and B. Z. Tang, Adv. Funct. Mater., 2017, 27, 1704039; (i) G.-F. Zhang, H. Wang, M. P. Aldred, T. Chen, Z.-Q. Chen, X. Meng and M.-Q. Zhu, Chem. Mater., 2014, 26, 4433-4446; (j) T. Jadhav, B. Dhokale, S. M. Mobin and R. Misra, J. Mater. Chem. C, 2015, 3, 9981-9988.

2 (a) F. He, Y. L. Tang, S. Wang, Y. L. Li and D. B. Zhu, J. Am. Chem. Soc., 2005, 127, 12343-12346; (b) M. T. Gabr and F. C. Pigge, Mater. Chem. Front., 2017, 1, 1654-1661.

3 (a) E. House, J. Collingwood, A. Khan, O. Korchazkina, G. Berthon and C. Exleya, J. Alzheimer's Dis., 2004, 6, 291-301; (b) G. Berthon, Coord. Chem. Rev., 2002, 228, 319-341.

4 (a) H. Arakawa, R. Ahmad, M. Naoui and H.-A. Tajmir-Riahi, J. Biol. Chem., 2000, 275, 10150-10153; (b) J. B. Vincent, Nutr. Rev., 2000, 58, 67-72.

5 (a) S. K. Sahoo, D. Sharma, R. K. Bera, G. Crisponi and J. F. Callan, Chem. Soc. Rev., 2012, 41, 7195-7227; (b) X. Yang, X. Chen, X. Lu, C. Yan, Y. Xu, X. Hang, J. Qu and R. Liu, J. Mater. Chem. C, 2016, 4, 383-390.

6 (a) G. Balamurugan, S. Velmathi, N. Thirumalaivasan and S. P. Wu, Analyst, 2017, 142, 4721-4726; (b) A. Anwara, A. Minhazc, N. A. Khana, K. Kalantari, A. B. M. Afifid and M. R. Shahb, Sens. Actuators, B, 2018, 257, 875-881; (c) H. Li, P. Zhang, L. P. Smaga, R. A. Hoffman and J. Chan, J. Am. Chem. Soc., 2015, 137, 15628-15631; (d) L. Li, B. Yu and T. You, Biosens. Bioelectron., 2015, 74, 263-269; (e) P. C. Dhara, A. Pal, P. Mohanty and B. Bag, Sens. Actuators, B, 2015, 219, 308-314; (f) A. K. Dwivedi, M. Pandeeswar and T. Govindaraju, ACS Appl. Mater. Interfaces, 2014, 6, 21369-21379.

7 (a) D. Ma, B. Li, Z. Cui, K. Liu, C. Chen, G. Li, J. Hua, B. Ma, Z. Shi and S. Feng, ACS Appl. Mater. Interfaces, 2016, 8, 24097-24103; (b) A. S. Klymchenko, Acc. Chem. Res., 2017, 50, 366-375; (c) E. A. Owens, M. Henary, G. E. Fakhri and H. S. Choi, Acc. Chem. Res., 2016, 49, 1731-1740; (d) S. Shanmugaraju and P. S. Mukherjee, Chem. Commun., 2015, 51, 16014-16032.

8 (a) L. Wang, W. Li, W. Zhi, D. Ye, Y. Wang, L. Ni and X. Bao, Dyes Pigm., 2017, 147, 357-363; (b) Q. Sun, W. Wang,
Z. Chen, Y. Yao, W. Zhang, L. Duan and J. Qian, Chem. Commun., 2017, 53, 6432-6435; (c) D. Karak, A. Banerjee, S. Lohar, A. Sahana, S. K. Mukhopadhyay, S. S. Adhikari and D. Das, Anal. Methods, 2013, 5, 169-172; (d) K. Gu, Y. Xu, H. Li, Z. Guo, S. Zhu, S. Zhu, P. Shi, T. D. James, H. Tian and W.-H. Zhu, J. Am. Chem. Soc., 2016, 138, 5334-5340.

9 (a) G. Sivaraman and D. Chellappa, J. Mater. Chem. B, 2013, 1, 5768-5772; (b) Z. Li, Z. Xue, Z. Wu, J. Han and S. Han, Org. Biomol. Chem., 2011, 9, 7652-7654; (c) M. Yu, R. Yuan, C. Shi, W. Zhou, L. Wei and Z. Li, Dyes Pigm., 2013, 99, 887-894; (d) H. N. Kim, M. H. Lee, H. J. Kim, J. S. Kim and J. Yoon, Chem. Soc. Rev., 2008, 37, 1465-1472.

10 M. Beija, C. A. M. Afonso and J. M. G. Martinho, Chem. Soc. Rev., 2009, 38, 2410-2433.

11 A. Sahana, A. Banerjee, S. Lohar, A. Banik, S. K. Mukhopadhyay, D. A. Safin, M. G. Babashkina, M. Bolte, Y. Garcia and D. Das, Dalton Trans., 2013, 42, 13311-13314.

12 (a) J. Y. Hu, J. Wang, R. Liu, Y. Li, J. P. Lu and H. J. Zhu, Dyes Pigm., 2018, 153, 84-91; (b) S.-W. Cheng, T. Han, T.-Y. Huang, Y.-H. C. Chien, C.-L. Liu, B. Z. Tang and G.-S. Liou, ACS Appl. Mater. Interfaces, 2018, 10, 18281-18288.

13 (a) L. Zong, Y. Xie, C. Wang, J.-R. Li, Q. Li and Z. Li, Chem. Commun., 2016, 52, 11496-11499; (b) D. Li, Y. Zhang, Z. Fan, J. Chen and J. Yu, Chem. Sci., 2015, 6, 6097-6101; (c) Y. Yuan and B. Liu, Chem. Sci., 2017, 8, 2537-2546; (d) M. Huang, R. Yu, K. Xu, S. Ye, S. Kuang, X. Zhu and Y. Wan, Chem. Sci., 2016, 7, 4485-4491; (e) S. Li, M. Gao, S. Wang, R. Hu, Z. Zhao, A. Qin and B. Z. Tang, Chem. Commun., 2017, 53, 4795-4798.

14 J. Luo, Z. Xie, J. W. Y. Lam, L. Cheng, H. Chen, C. Qiu, H. S. Kwok, X. Zhan, Y. Liu, D. Zhu and B. Z. Tang, Chem. Commun., 2001, 1740-1741.

15 (a) S. Chen, Q. Chen, Q. Li, J. An, P. Sun, J. Ma and H. Gao, Chem. Mater., 2018, 30, 1782-1790; (b) W. Qin, Z. Yang, Y. Jiang, J. W. Y. Lam, G. Liang, H. S. Kwok and B. Z. Tang, Chem. Mater., 2015, 27, 3892-3901; (c) S. Tian, G. Liu, X. Wang, T. Wu, J. Yang, X. Ye, G. Zhang, J. Hu and S. Liu, ACS Appl. Mater. Interfaces, 2016, 8, 3693-3702; (d) G. Liang, F. Ren, H. Gao, F. Zhu, Q. Wu and B. Z. Tang, J. Mater. Chem. $A$, 2017, 5, 2115-2122; (e) H. Liu and H. Liu, J. Mater. Chem. A, 2017, 5, 9156-9162.

16 M. Shyamal, P. Mazumdar, S. Maity, G. P. Sahoo, G. SalgadoMorán and A. Misra, J. Phys. Chem. A, 2016, 120, 210-220.

17 S. Park, W. Kimb, K. M. K. Swamy, H. Y. Lee, J. Y. Jung, G. Kima, Y. Kima, S.-J. Kima and J. Yoon, Dyes Pigm., 2013, 99, 323-328.

18 (a) M. Gao, Y. Li, X. Chen, S. Li, L. Ren and B. Z. Tang, ACS Appl. Mater. Interfaces, 2018, 10, 14410-14417; (b) Y. Cheng, J. Dai, C. Sun, R. Liu, T. Zhai, X. Lou and F. Xia, Angew. Chem., Int. Ed., 2018, 57, 3123-3127.

19 (a) L. Wang, Y. Shen, Q. Zhu, W. Xu, M. Yang, H. Zhou, J. Wu and Y. Tian, J. Phys. Chem. C, 2014, 118, 8531-8540; (b) M. Shyamal, S. Maity, P. Mazumdar, G. P. Sahoo, R. Maity and A. Misra, J. Photochem. Photobiol., A, 2017, 342, 1-14; (c) L. Wang, Y. Shen, Q. Zhu, W. Xu, M. Yang, H. Zhou, J. Wu and Y. Tian, J. Phys. Chem. C, 2014, 118, 8531-8540. 
20 (a) A. J. Weerasinghe, C. Schmiesing, S. Varaganti, G. Ramakrishna and E. Sinn, J. Phys. Chem. B, 2010, 114, 9413-9419; (b) W. Lin, L. Long, L. Yuan, Z. Cao and J. Feng, Anal. Chim. Acta, 2009, 634, 262-266; (c) N. Narayanaswamy and T. Govindaraju, Sens. Actuators, B, 2012, 161, 304-310; (d) J. Wang, Y. Fan, H.-W. Lee, C. Yi, C. Cheng, X. Zhao and M. Yang, ACS Appl. Nano Mater., 2018, 1, 3747-3753; (e) Y. Yang, X. Song, C. Xu, Y. Wang, G. Zhang and W. Liu, Dalton Trans., 2018, 47, 11077-11083; $(f)$ L.-M. Liu and Z.-Y. Yang, Inorg. Chim. Acta, 2018, 469, 588-592; (g) H. J. Jang, J. H. Kang, D. Yun and C. Kim, J. Fluoresc., 2018, 28, 785-794; (h) H. Xu, M. Fang, C.-S. Cao, W.-Z. Qiao and B. Zhao, Inorg. Chem., 2016, 55, 4790-4794; (i) S. Zeng, S.-J. Li, X.-J. Sun, M.-Q. Li, Y.-Q. Ma, Z.-Y. Xing and J.-L. Li, Spectrochim. Acta, Part A, 2018, 205, 276-286; (j) M.-C. Tu, D. Rajwar, G. Ammanath, P. Alagappan, U. H. Yildiz and B. Liedberg, Anal. Chim. Acta, 2016, 912, 105-110; (k) X. Huang, C. Fan, Z. Wang, X. Zhan, M. Pei and Z. Lu, Inorg. Chem. Commun., 2015, 57, 62-65; (l) T. Rasheed, C. Li, L. Fu, F. Nabeel, C. Yu, L. Gong and Y. Zhou, J. Mol. Liq., 2018, 272, 440-449; ( $m$ ) H. Jin, J. Xu, L. Zhang, B. Ma, X. Shi, Y. Fan and L. Wang, J. Solid State Chem., 2018, 268, 168-174; (n) V. Bravo, S. Gil, A. M. Costero, M. N. Kneeteman, U. Llaosa, P. M. E. Mancini, L. E. Ochando and M. Parra, Tetrahedron, 2012, 68, 4882-4887; (o) N. Manjubaashini, T. D. Thangadurai, G. Bharathi and D. Nataraj, J. Fluoresc., 2018, 202, 282-288.

21 H. N. Kim, M. H. Lee, H. J. Kim, J. S. Kim and J. Yoon, Chem. Soc. Rev., 2008, 37, 1465-1472.

22 X. Chen, T. Pradhan, F. Wang, J. S. Kim and J. Yoon, Chem. Rev., 2012, 112, 1910-1956.
23 X. Chen, X. Y. Shen, E. Guan, Y. Liu, A. Qin, J. Z. Sun and B. Z. Tang, Chem. Commun., 2013, 9, 1503-1505.

24 X. Zeng, L. Dong, C. Wu, L. Mu, S.-F. Xu and Z. Tao, Sens. Actuators, B, 2009, 141, 506-510.

25 (a) H. Sun, S. Liu, W. Lin, K. Y. Zhang, W. Lv, X. Huang, F. Huo, H. Yang, G. Jenkins, Q. Zhao and W. Huang, Nat. Commun., 2014, 5, 4601; (b) A. Kishimura, T. Yamashita, K. Yamaguchi and T. Aida, Nat. Mater., 2005, 4, 546-549; (c) T. Mutai, H. Satou and K. Araki, Nat. Mater., 2005, 4, 685-687; (d) Y. Wu, Y. Xie, Q. Zhang, H. Tian, W. Zhu and A. D. Q. Li, Angew. Chem., Int. Ed., 2014, 53, 2090-2094; (e) D. J. Wales, Q. Cao, K. Kastner, E. Karjalainen, G. N. Newton and V. Sans, Adv. Mater., 2018, 30, 1800159; $(f)$ T. Butler, W. A. Morris, J. Samonina-Kosicka and C. L. Fraser, ACS Appl. Mater. Interfaces, 2016, 8, 1242-1251; (g) Z. Song, R. Liu, Y. Li, H. Shi, J. Hu, X. Cai and H. Zhu, J. Mater. Chem. C, 2016, 4, 2553-2559; (h) Y. Li, L. Chen, Y. Ai, E. Y.-H. Hong, A. K.-W. Chan and V. W.-W. Yam, J. Am. Chem. Soc., 2017, 139, 13858-13866; (i) S. Mo, Q. Meng, S. Wan, Z. Su, H. Yan, B. Z. Tang and M. Yin, Adv. Funct. Mater., 2017, 27, 1701210; (j) C. Wang and Z. Li, Mater. Chem. Front., 2017, 1, 2174-2194. 26 (a) P. Xue, J. Ding, Y. Shen, H. Gao, J. Zhao, J. Sun and R. Lu, J. Mater. Chem. C, 2017, 5, 11532-11541; (b) M. E. Genovese, A. Athanassiou and D. Fragouli, J. Mater. Chem. A, 2015, 3, 22441-22447; (c) F. Nisic, A. Colombo, C. Dragonetti, M. Fontani, D. Marinotto, S. Righetto, D. Roberto and J. A. G. Williams, J. Mater. Chem. C, 2015, 3, 7421-7427.

27 (a) D. D. La, S. V. Bhosale, L. A. Jones and S. V. Bhosale, ACS Appl. Mater. Interfaces, 2018, 10, 12189-12216; (b) N. N. Li, J. Z. Li, P. Liu, D. Pranantyo, L. Luo, J. C. Chen, E.-T. Kang, X. F. Hu, C. M. Li and L. Q. Xu, Chem. Commun., 2017, 53, 3315-3318. 